\title{
Predictors of Recurrence and Survival in Malignant Triton Tumours: A Case Report and Systematic Review Systematic Review of Malignant Triton Tumours
}

\author{
Allison Elizabeth Pumputis \\ Schulich School of Medicine and Dentistry, Western University https://orcid.org/0000-0002-2451-9531 \\ Laura Callan \\ Department of Radiation Oncology, Western University \\ Anna Branch \\ Schulich School of Medicine and Dentistry, Western University \\ R. Gabriel Boldt \\ London Regional Cancer Program, London Health Sciences Centre \\ Andrew Warner \\ Department of Radiation Oncology, Western University \\ Diane Logan \\ Department of Medical Oncology, Western University \\ Nancy Read \\ Department of Radiation Oncology, Western University \\ Fawaz Siddiqi \\ Department of Clinical Neurological Sciences, Western University \\ Douglas Ross \\ Division of Plastic Surgery, Department of Surgery, Western University \\ Luc Dubois \\ Division of Vascular Surgery, Department of Surgery, Western University \\ Dalilah Fortin \\ Division of Thoracic Surgery, Department of Surgery, Western University \\ David A. Palma \\ Department of Radiation Oncology, Western University \\ Anthony C. Nichols ( $\square$ Anthony.Nichols@lhsc.on.ca) \\ Department of Otolaryngology - Head and Neck Surgery, Western University https://orcid.org/0000-0002-0760-980X
}

Research

Keywords: Malignant triton tumour, malignant peripheral nerve sheath tumours, surgery, radiotherapy, outcomes

Posted Date: January 15th, 2021

DOI: https://doi.org/10.21203/rs.3.rs-144663/v1

License: (a) (1) This work is licensed under a Creative Commons Attribution 4.0 International License. Read Full License 


\section{Abstract}

Background: Malignant triton tumours (MTTs) are a rare and aggressive subset of malignant peripheral nerve sheath tumours (MPNSTs). A systematic review was conducted to better understand the prognosis and prognostic factors of this tumour in adult patients, and to provide treatment guidance for clinicians encountering this rare tumour.

Methods: A PubMed search was conducted using the keywords "rhabdomyoblastic," "rhabdomyosarcomatous," "triton," "case series" and "case report". A reference list search was also completed. Two independent investigators completed abstract and full-text reviews. Articles were restricted to peer-reviewed articles in English language only containing adult MTT cases ( $\geq 18$ years), and excluding articles with insufficient treatment/outcome data. Univariable and multivariable Cox proportional hazards regression was performed to identify significant predictors of overall survival (OS) and progression-free survival (PFS).

Results: A total of 123 cases from the literature and 1 case from our institution were included in the final analysis. The 2-year and 5-year OS was $46.2 \%$ and $32.2 \%$, and the 2-year and 5-year PFS was $27.1 \%$ and $21.3 \%$, respectively. On multivariable analysis for OS, prior radiation exposure (hazard ratio [HR]: $3.99, p=0.027)$, central nervous system or spine disease site $(H R: 5.86, p<0.001)$ and positive neurofibromatosis 1 (NF1) status (HR: $3.42, p<0.001$ ) were associated with worse survival. Adjuvant radiotherapy (HR: $0.58, p=0.038)$ was associated with improved survival. Positive NF1 status (HR: 2.34, $p<0.001)$ and positive margins (HR: $3.28, p<0.001)$ were associated with worse PFS.

Conclusion: MTTs are rare and have poor long-term survival. Negative surgical margins and adjuvant radiation were found to be associated with improved outcomes.

\section{Background}

Malignant triton tumours (MTTs) are a very rare subset of malignant peripheral nerve sheath tumours (MPNSTs) (1). MPNSTs are classified as MTTs when there is presence of rhabdomyoblastic differentiation, identified by immunohistochemical staining positive for MPNST and rhabdomyoblastic markers (2). MTTs were first identified in 1932 by Masson et al., and were termed "triton tumours" in 1973 by Woodruff et al.(3) They are aggressive tumours with 5 -year survival rates of $14 \%-35 \%(1,4)$. Risk factors include a history of neurofibromatosis 1 (NF1) and previous radiation exposure(1,5). The most common tumour sites reported are within the head and neck, and extremities $(2,5)$. Prior retrospective reports have suggested that patients with head and neck MTTs experience improved survival compared with other sites $(2,5)$.

There is no uniform consensus on treatment strategy for MTTs(5). There have been several treatment recommendations in the literature including radical surgical excision with negative margins, and the adjuvant use of radiotherapy and chemotherapy. However, these recommendations have been based on limited evidence. The goal of this study was to systematically review the literature to provide guidance on the optimal treatment regimen, and to identify prognostic factors for this rare and aggressive tumour. We also present a case of a 45-year-old man with a MTT located in the neck invading the spine, brachial plexus and upper mediastinum.

\section{Case Report}

A 45-year-old previously healthy man presented with a 1-year history of a left neck mass and intermittent left arm paraesthesia. On physical examination, the patient had intact cranial nerve function and normal strength, sensation and range of motion in the upper and lower extremities bilaterally with no long-tract findings. The patient had no personal or family history of or any clinical signs of NF1. Ultrasound and magnetic resonance imaging (MRI) showed a solid mass with a complex cystic appearance $(5.7 \mathrm{~cm} \times 7.5 \mathrm{~cm} \times 8.7 \mathrm{~cm})$ arising from the left brachial plexus with predominant involvement of the lower trunk (Fig. 1A), encasing the left subclavian artery and vein, left vertebral artery, innominate vein, and left thyrocervical trunk and involving the clavicle, first rib and C7 and T1 vertebral bodies (Figs. 1B and 1C). There was also questionable involvement of the vagus and phrenic nerves.

An open biopsy revealed a spindle cell lesion with focal rhabdomyoblasts. The lesion stained positive for desmin and myogen and showed loss of H3K27 trimethylation. These findings supported a diagnosis of a MPNST with rhabdomyoblastic differentiation, in keeping with MTT. This was confirmed on repeat biopsy.

The case was discussed at a provincial sarcoma multidisciplinary tumour board involving surgery, medical oncology, radiation oncology, radiology and pathology from multiple academic centres. Based on this discussion, the importance of R0 resection (resection with negative margins) was emphasized and a plan for maximal resection followed by post-operative radiotherapy was made. 
Due to the location of the tumour, five surgical teams were involved in the surgical planning and execution. The surgery was led by a head and neck oncologic surgeon (ACN), with vascular surgery, thoracic surgery, spine surgery and plastic surgery involved. Due to the involvement of the left vertebral artery, the patient underwent a balloon-test occlusion and pre-operative embolization of that vessel. The subsequent resection involved a left neck dissection, resection of the left clavicle, left upper sternum, the left apical pleura and partial corpectomies of the C5-T1 vertebrae and the entire brachial plexus (Fig. 2A). An anterior cervical spine reconstruction and fusion was carried out. The subclavian artery was grafted with an $8 \mathrm{~mm}$ Gortex ${ }^{\circledR}$ interposition graft. The brachial plexus was reconstructed using the left medial and lateral antebrachial cutaneous nerves to reconstruct the axillary nerve, biceps motor branch and spinal accessory nerves using $\mathrm{C} 7$ as the proximal source of axons. Because of the extent of proximal nerve involvement, the other cervical roots were not available. A pectoralis major flap was used to cover the resection site. The total operative time was 15 hours. The patient had an estimated blood loss of $7 \mathrm{~L}$ and required 8 units of packed red blood cells, 4 units of fresh frozen plasma, 1-unit cryoprecipitate, $1 \mathrm{~L}$ of $5 \%$ albumin, and $200 \mathrm{~mL}$ of $25 \%$ albumin. The patient was extubated on post-operative day one with no complications.

Pathology confirmed a grade 3 MTT with epithelioid and spindle cell components measuring $5.5 \mathrm{~cm} \times 4.5 \mathrm{~cm} \times 3.9 \mathrm{~cm}$ (Fig. 2B). The left pleural margin was initially positive, however, further resection of the pleura in this area cleared the margin. None of the 24 lymph nodes were involved ( $\mathrm{pT}$ 4NOMO). The patient received adjuvant external beam radiation. The pre-operative CT and MRI scans were fused to aid in target delineation. Sixty Gy in 30 daily fractions was prescribed to the entire pre-operative tumour volume and surgical bed, with a simultaneous in-field boost to the pleural margin to $64 \mathrm{~Gy}$. The treatment was planned and delivered using volumetric arc therapy with daily cone-beam CT image guidance. The patient tolerated the treatment well, with grade 2 erythema and grade 1 esophagitis (Common Terminology Criteria for Adverse Events [CTCAE] version 5.0).

As expected, following surgery the patient had a complete brachial plexus palsy, a left-sided Horner's syndrome and left phrenic nerve paralysis. At last follow-up, 14 months from the time of surgery, the patient is alive and free of disease. He is able to shoulder shrug but, as expected given the normal rates of nerve recovery, there are no motor units in either biceps or deltoid detected on electrodiagnostic testing. Despite this, he has returned to work as well as sporting activities including playing hockey as a goalie and baseball with the aid of assistive devices.

\section{Methods}

A PubMed search was completed using the keywords "rhabdomyoblastic," "rhabdomyosarcomatous," "triton," "case series" and "case report", with no limits set on the search. Refer to Additional File 1 for the full electronic search strategy. Following PRISMA guidelines, two investigators (AEP and LC) independently completed abstract reviews eliminating any studies that were not in English language or that did not involve MTTs. Investigators then searched through the reference lists of the remaining articles for further relevant studies. The remaining articles along with articles identified from the reference search, underwent full-text review independently by two investigators (AEP and $A B$ ) to create two independent datasets. The final dataset was created by discussing any discrepancies between the two datasets, and involving a third investigator (LC) to help reach consensus when required. Articles met inclusion criteria if they were peer-reviewed and contained adult case(s) of MTTs with treatment and outcome information. Articles were eliminated if they were not in English language, did not contain cases of MTTs, reported on cases of paediatric MTTs only (under the age of 18 years), or were missing treatment or outcome information. The risk of bias within individual articles was not assessed as the articles included were case reports.

Demographic and tumour characteristics, treatment regimens, current status and timing of disease progression were collected. Case demographic characteristics included: age, sex, NF1 status, and any previous radiation exposure. If previous radiation exposure was not stated, it was assumed to be negative. Tumour characteristics included: tumour site, size, grade and stage (determined using in-text description based on the American Joint Committee on Cancer [AJCC] 8th edition), mitotic rate (high: $\geq 20$ mitotic figures per 10 high power field [HPF]; low: < 20 mitotic figures per $10 \mathrm{HPF}$ ), histologic staining, and any divergent differentiation noted. Data on treatment regimens included: surgical resection and margin status, radiation dose and fractionation, and chemotherapy regimen for the primary tumour and/or any recurrent disease. Case status and time to recurrence or death (if applicable) and last follow-up were also collected.

\section{Statistical Analysis:}

Descriptive statistics and Kaplan-Meier estimates for overall survival (OS) and progression-free survival (PFS) were generated for all cases. OS and PFS were calculated from date of treatment to date of progression (PFS only), date of death due to any cause, or date of last follow-up, whichever occurred first. Univariable and multivariable Cox proportional hazards regressions were performed for OS and PFS. All eligible variables with univariable $p$-values $<0.05$ and available in more than $50 \%$ of cases were incorporated into a multivariable

Page $3 / 15$ 
regression model and sequentially removed using backward elimination techniques until all remaining covariates had $p$-values $<0.05$.

Kaplan-Meier estimates were additionally generated for all factors remaining significant on multivariable analysis. All statistical analysis was completed using SAS version 9.4 software (SAS Institute, Cary, NC, USA), using two-sided statistical testing at the 0.05 significance level.

\section{Results}

\section{Manuscript Review}

A total of 285 articles, published between 1976 and 2019, were identified from PubMed search. The search was last completed on October 24th, 2019. One-hundred-sixty-eight articles were eliminated during abstract review. Twelve additional articles were identified from reference list search. A total of 129 articles underwent full-text review. Forty-one articles were eliminated during full-text review, leaving 88 articles reporting on 123 independent cases to be included in the final analysis. The abovementioned case was also included, for a total of 124 cases of adult MTTs (Fig. 3). Refer to Additional File 2 for a list of articles used in the statistical analysis.

\section{Case Demographics and Treatment Details}

Case demographics and treatment details are summarized in Table 1. The mean \pm SD age was $42.8 \pm 17.6$ years and 76 (62.8\%) of the cases were male. The mean age for male cases was $41.4 \pm 17.3$ years compared to $44.6 \pm 17.4$ years for female cases. NF1 status was reported in 103 cases, of which $36(35.0 \%)$ of the cases were positive and the majority were male $(n=26,72.2 \%)$. Among those with positive NF1 status, mean age was $35.2 \pm 12.2$ years and $37.8 \pm 12.6$ years for male and female cases, respectively. Divergent differentiation was present in $24(19.8 \%)$ of the 121 cases in which it was reported. The most common types were chondroid $(n=5$, $20.8 \%)$, osteocartilaginous $(n=5,20.8 \%)$ and glandular $(n=4,16.7 \%)$. Previous radiation exposure was reported in 5 cases $(4.0 \%)$. The most common tumour sites were head and neck $(n=35,28.2 \%)$, extremity $(n=25,20.2 \%)$ and trunk $(n=23,18.6 \%)$. Most cases received surgery $(n=118,95.9 \%)$ and radiotherapy $(n=74,60.7 \%)$. Chemotherapy was received by a minority of cases $(n=43,35.5 \%)$, and two $(1.6 \%)$ received no treatment. 


\begin{tabular}{|c|c|c|}
\hline Characteristic & $\mathbf{N}$ & All Cases $(n=124)$ \\
\hline Age - mean $\pm S D$ & 124 & $42.8 \pm 17.6$ \\
\hline Male - $\mathrm{n}(\%)$ & 121 & $76(62.8)$ \\
\hline \multicolumn{3}{|l|}{ Site - n(\%) } \\
\hline Head and neck & 124 & $35(28.2)$ \\
\hline Trunk & & $23(18.6)$ \\
\hline Extremities & & $25(20.2)$ \\
\hline Abdomen & & $3(2.4)$ \\
\hline Thorax & & $12(9.7)$ \\
\hline Retroperitoneum & & $10(8.1)$ \\
\hline Central nervous system & & $10(8.1)$ \\
\hline Spine & & $6(4.8)$ \\
\hline \multicolumn{3}{|l|}{ T stage - n(\%) } \\
\hline T1 & 110 & $9(8.2)$ \\
\hline T2 & & $20(18.2)$ \\
\hline T3 & & $33(30.0)$ \\
\hline T4 & & $8(7.3)$ \\
\hline T4a & & $33(30.0)$ \\
\hline $\mathrm{T} 4 \mathrm{~b}$ & & $6(5.5)$ \\
\hline T4c & & $1(0.9)$ \\
\hline \multicolumn{3}{|l|}{ N stage - n(\%) } \\
\hline NO & 113 & 109 (96.5) \\
\hline N1 & & $4(3.5)$ \\
\hline \multicolumn{3}{|l|}{ M stage - n(\%) } \\
\hline MO & 113 & $112(99.1)$ \\
\hline M1 & & $1(0.9)$ \\
\hline \multicolumn{3}{|l|}{ Treatment - n(\%) } \\
\hline None & 123 & $2(1.6)$ \\
\hline Surgery only & & $48(39.0)$ \\
\hline Surgery with adjuvant RT & & $42(34.2)$ \\
\hline Surgery with adjuvant CT & & $11(8.9)$ \\
\hline Surgery with adjuvant CRT & & $13(10.6)$ \\
\hline Other & & $7(5.7)$ \\
\hline
\end{tabular}

SD - Standard deviation; RT - radiotherapy; CT - chemotherapy; CRT - chemoradiotherapy; NF1 - neurofibromatosis 1; HPF - high power fields; $\mathrm{Cl}$ - confidence interval; ${ }^{1}$ Reverse Kaplan-Meier Method 


\begin{tabular}{|c|c|c|}
\hline Characteristic & $\mathbf{N}$ & All Cases $(n=124)$ \\
\hline Maximum size $(\mathrm{cm})-$ mean $\pm S D$ & 92 & $9.5 \pm 5.7$ \\
\hline Surgery - n(\%) & 123 & $118(95.9)$ \\
\hline Chemotherapy - n(\%) & 121 & $43(35.5)$ \\
\hline Radiotherapy - n(\%) & 122 & $74(60.7)$ \\
\hline \multicolumn{3}{|l|}{ Margins - n(\%) } \\
\hline Negative & 124 & $43(34.7)$ \\
\hline Positive & & $28(22.6)$ \\
\hline Unknown & & $53(42.7)$ \\
\hline NF1 status - n(\%) & 103 & $36(35.0)$ \\
\hline Divergent differentiation- n(\%) & 121 & $24(19.8)$ \\
\hline \multicolumn{3}{|l|}{ Mitoses - n(\%) } \\
\hline Low ( $\leq 20$ per HPF) & 124 & $16(12.9)$ \\
\hline High (> 20 per HPF) & & $20(16.1)$ \\
\hline Unknown & & $88(71.0)$ \\
\hline \multicolumn{3}{|l|}{ Grade - n(\%) } \\
\hline Low & 124 & $12(9.7)$ \\
\hline High & & $31(25.0)$ \\
\hline Unknown & & $81(65.3)$ \\
\hline Previous radiation exposure - $n(\%)$ & 124 & $5(4.0)$ \\
\hline \multicolumn{3}{|l|}{ Status - n(\%) } \\
\hline Death & 124 & $72(58.1)$ \\
\hline Alive with disease & & $9(7.3)$ \\
\hline Alive without disease & & $37(29.8)$ \\
\hline Alive unknown status & & $6(4.8)$ \\
\hline Recurrence - n(\%) & 124 & $63(50.8)$ \\
\hline Local recurrence - n(\%) & 124 & $37(29.8)$ \\
\hline Regional recurrence - n(\%) & 124 & $4(3.2)$ \\
\hline Distant recurrence - n(\%) & 124 & 35 (28.2) \\
\hline
\end{tabular}

SD - Standard deviation; RT - radiotherapy; CT - chemotherapy; CRT - chemoradiotherapy; NF1 - neurofibromatosis 1; HPF - high power fields; $\mathrm{Cl}$ - confidence interval; ${ }^{1}$ Reverse Kaplan-Meier Method 


\begin{tabular}{|c|c|c|}
\hline Characteristic & $\mathbf{N}$ & All Cases $(n=124)$ \\
\hline \multicolumn{3}{|l|}{ Recurrence pattern - $n(\%)$} \\
\hline Local & 63 & $24(38.1)$ \\
\hline Regional & & $3(4.8)$ \\
\hline Distant & & $23(36.5)$ \\
\hline Local and distant & & $12(19.1)$ \\
\hline Local and regional & & $1(1.6)$ \\
\hline Recurrence or death - $\mathrm{n}(\%)$ & 124 & $85(68.6)$ \\
\hline Median follow-up (years) ${ }^{1}-$ median $(95 \% \mathrm{Cl})$ & 124 & $3.8(2.5,5.0)$ \\
\hline
\end{tabular}

\section{Outcomes and Predictors of Survival}

With a median follow-up time of 3.8 years (95\% confidence interval [Cl]: 2.5-5.0), 63 cases (50.8\%) had known recurrent disease and over half of the cases were deceased $(n=72,58.1 \%)$. The majority of recurrences were local $(n=37,58.7 \%)$, although distant recurrences occurred almost as frequently $(n=35,55.6 \%)$. Median, 2-year, and 5-year OS was 22 months, $46.2 \%$ and 32.2\%, and the median, 2-year, and 5-year PFS was 8 months, $27.1 \%$ and $21.3 \%$, respectively (Fig. 4 A and B).

Results from univariable and multivariable Cox proportional hazards regression are summarized in Table 2. Univariable analysis identified NF1 status (hazard ratio [HR]: 3.45; 95\% Cl: 2.04-5.83; $p<0.001$ ), previous radiation exposure (HR: 2.96; 95\% Cl: 1.18-7.46; $p$ $=0.021$ ), central nervous system (CNS) or spine vs. other tumour site (HR: 5.38; 95\% Cl: $2.79-10.37 ; p<0.001)$, positive vs. negative margin status (HR: 2.28; 95\% Cl: 1.22-4.24; $p=0.009)$, high vs. low grade (HR: $6.21 ; 95 \% \mathrm{Cl}: 1.44-26.87 ; p=0.015)$, high vs. low mitoses (HR: $5.17 ; 95 \% \mathrm{Cl}: 1.48-18.02 ; \mathrm{p}=0.010$ ), and divergent differentiation (HR: $1.83 ; 95 \% \mathrm{Cl}: 1.08-3.10 ; p=0.025)$ as significant predictors of worse OS and radiotherapy (HR: $0.57 ; 95 \% \mathrm{Cl}: 0.36-0.91 ; \mathrm{p}=0.019$ ) as a significant predictor of improved OS. On multivariable analysis, CNS or spine tumour site (HR: 5.86; $95 \%$ Cl: $2.82-12.20 ; p<0.001$ ), radiotherapy (HR: 0.58; $95 \%$ Cl: $0.35-0.97 ; p=0.038$ ), NF1 status (HR: $3.42 ; 95 \% \mathrm{Cl}: 1.99-5.88 ; \mathrm{p}<0.001$ ), and prior radiation exposure (HR: $3.99 ; 95 \% \mathrm{Cl}: 1.17-13.54 ; \mathrm{p}=0.027$ ) remained significant predictors of OS. 
Table 2

Univariable and multivariable Cox proportional hazards regression models for all cases $(n=124)$.

\begin{tabular}{|c|c|c|c|c|}
\hline \multirow{2}{*}{$\begin{array}{l}\text { Dependent Variable: } \\
\text { Variable: }\end{array}$} & \multicolumn{2}{|l|}{ Overall Survival } & \multicolumn{2}{|c|}{ Progression-Free Survival } \\
\hline & $\mathrm{HR}(95 \% \mathrm{Cl})$ & p-value & $\mathrm{HR}(95 \% \mathrm{Cl})$ & p-value \\
\hline \multicolumn{5}{|l|}{ Univariable: } \\
\hline Age (per 5 years) & $0.99(0.93,1.05)$ & 0.660 & $1.00(0.94,1.06)$ & 0.950 \\
\hline Male (vs. female) & $1.40(0.84,2.31)$ & 0.195 & $1.38(0.87,2.20)$ & 0.177 \\
\hline Site (vs. head and neck) ${ }^{1}$ & & $<0.001$ & & 0.320 \\
\hline Trunk & $1.57(0.77,3.21)$ & 0.217 & $1.11(0.58,2.13)$ & 0.759 \\
\hline Extremities & $1.35(0.67,2.72)$ & 0.401 & $1.21(0.64,2.29)$ & 0.562 \\
\hline \multirow[t]{4}{*}{ Abdomen } & $0.91(0.21,3.91)$ & 0.893 & $0.76(0.18,3.25)$ & 0.716 \\
\hline & $1.41(0.52,3.84)$ & 0.500 & $1.24(0.57,2.70)$ & 0.582 \\
\hline & $3.26(1.34,7.96)$ & 0.009 & $1.94(0.82,4.61)$ & 0.132 \\
\hline & $9.28(3.85,22.39)$ & $<0.001$ & $2.65(1.20,5.85)$ & 0.016 \\
\hline Thorax & $5.44(1.78,16.58)$ & 0.003 & $1.81(0.62,5.28)$ & 0.277 \\
\hline \multicolumn{5}{|l|}{ Retroperitoneum } \\
\hline \multicolumn{5}{|l|}{ Central nervous system } \\
\hline \multicolumn{5}{|l|}{ Spine } \\
\hline Trunk or extremities (yes vs. no) & $0.89(0.55,1.43)$ & 0.617 & $0.90(0.58,1.40)$ & 0.647 \\
\hline Thorax or abdomen (yes vs. no) & $0.76(0.35,1.66)$ & 0.491 & $0.89(0.47,1.68)$ & 0.720 \\
\hline Central nervous system or spine (yes vs. no) & $5.38(2.79,10.37)$ & $<0.001$ & $2.00(1.10,3.66)$ & 0.024 \\
\hline T stage & & 0.496 & & 0.499 \\
\hline T3 vs. T1-T2 & $1.40(0.70,2.77)$ & 0.340 & $1.18(0.63,2.21)$ & 0.610 \\
\hline T4 vs. T1-T2 & $1.45(0.77,2.76)$ & 0.253 & $1.40(0.79,2.47)$ & 0.247 \\
\hline N stage (N1 vs. N0) & $1.66(0.52,5.31)$ & 0.392 & $1.46(0.46,4.65)$ & 0.521 \\
\hline Maximum size (per $5 \mathrm{~cm}$ ) & $1.06(0.85,1.32)$ & 0.610 & $1.08(0.89,1.31)$ & 0.422 \\
\hline Surgery (yes vs. no) & $0.86(0.27,2.75)$ & 0.797 & $0.61(0.22,1.66)$ & 0.331 \\
\hline Chemotherapy (yes vs. no) & $1.31(0.81,2.11)$ & 0.271 & $1.69(1.09,2.63)$ & 0.019 \\
\hline Radiotherapy (yes vs. no) & $0.57(0.36,0.91)$ & 0.019 & $0.65(0.42,1.00)$ & 0.048 \\
\hline Margins (vs. negative) & & 0.027 & & 0.002 \\
\hline Positive & $2.28(1.22,4.24)$ & 0.009 & $2.79(1.58,4.94)$ & $<0.001$ \\
\hline Unknown & $1.79(1.02,3.15)$ & 0.042 & $1.72(1.02,2.90)$ & 0.043 \\
\hline NF1 status (yes vs. no) & $3.45(2.04,5.83)$ & $<0.001$ & $2.81(1.72,4.58)$ & $<0.001$ \\
\hline Divergent (yes vs. no) & $1.83(1.08,3.10)$ & 0.025 & $1.52(0.92,2.52)$ & 0.104 \\
\hline Mitoses (vs. low ( $\leq 20$ per HPF) $)^{1}$ & & 0.026 & & 0.062 \\
\hline High (>20 per HPF) & $5.17(1.48,18.02)$ & 0.010 & $2.91(1.18,7.14)$ & 0.020 \\
\hline Unknown & $4.85(1.51,15.54)$ & 0.008 & $2.29(1.05,5.01)$ & 0.038 \\
\hline
\end{tabular}

HR - Hazard Ratio; Cl - Confidence interval; NF1 - neurofibromatosis 1; HPF - high power field; P-values < 0.05 shown in BOLD; ${ }^{1}$ Excluded from multivariable modelling 


\begin{tabular}{|c|c|c|c|c|}
\hline \multirow{2}{*}{$\begin{array}{l}\text { Dependent Variable: } \\
\text { Grade (vs. low) }{ }^{1}\end{array}$} & \multirow[t]{2}{*}{ Overall Survival } & \multicolumn{3}{|c|}{ Progression-Free Survival } \\
\hline & & 0.036 & & 0.094 \\
\hline High & $6.21(1.44,26.87)$ & 0.015 & $2.41(0.90,6.45)$ & 0.080 \\
\hline Unknown & $6.42(1.56,26.44)$ & 0.010 & $2.77(1.10,6.96)$ & 0.030 \\
\hline Radiation exposure (yes vs. no) & $2.96(1.18,7.46)$ & 0.021 & $2.20(0.89,5.48)$ & 0.089 \\
\hline Necrosis (vs. no) ${ }^{1}$ & & 0.084 & & 0.251 \\
\hline Yes & $2.47(0.75,8.06)$ & 0.136 & $2.66(0.82,8.65)$ & 0.105 \\
\hline Unknown & $1.55(0.48,5.04)$ & 0.469 & $2.67(0.83,8.57)$ & 0.100 \\
\hline Haemorrhage (vs. no) ${ }^{1}$ & & 0.370 & & 0.970 \\
\hline Yes & $1.00(0.22,4.48)$ & 0.994 & $0.85(0.19,3.77)$ & 0.828 \\
\hline Unknown & $0.65(0.16,2.69)$ & 0.555 & $0.90(0.22,3.67)$ & 0.879 \\
\hline \multicolumn{5}{|l|}{ Multivariable: } \\
\hline Central nervous system or spine (yes vs. no) & $5.86(2.82,12.20)$ & $<0.001$ & - & - \\
\hline Radiotherapy (yes vs. no) & $0.58(0.35,0.97)$ & 0.038 & - & - \\
\hline Margins (vs. negative) & & - & & 0.002 \\
\hline Positive & - & - & $3.28(1.72,6.28)$ & $<0.001$ \\
\hline Unknown & - & - & $1.66(0.94,2.95)$ & 0.083 \\
\hline NF1 (yes vs. no) & $3.42(1.99,5.88)$ & $<0.001$ & $2.34(1.42,3.85)$ & $<0.001$ \\
\hline Radiation exposure (yes vs. no) & $3.99(1.17,13.54)$ & 0.027 & - & - \\
\hline
\end{tabular}

Similarly for PFS, univariable analysis identified NF1 status (HR: $2.81 ; 95 \% \mathrm{Cl}: 1.72-4.58 ; \mathrm{p}<0.001)$, CNS or spine tumour sites (HR: 2.00; 95\% Cl: $1.10-3.66 ; p=0.024)$, positive margin status (HR: 2.79; 95\% Cl: 1.58-4.94; $p<0.001$ ), chemotherapy (HR: $1.69 ; 95 \%$ Cl: $1.09-$ 2.63; $p=0.019$ ), and high mitoses (HR: $2.91 ; 95 \% \mathrm{Cl}: 1.18-7.14 ; \mathrm{p}=0.020$ ) as significant predictors of worse PFS and radiotherapy (HR: $0.65 ; 95 \% \mathrm{Cl}: 0.42-1.00 ; \mathrm{p}=0.048$ ) as a significant predictor of improved PFS. Positive margin status (HR: $3.28 ; 95 \% \mathrm{Cl}: 1.72-6.28 ; \mathrm{p}<$ 0.001 ) and NF1 status (HR: 2.34; $95 \%$ Cl: $1.42-3.85 ; p<0.001)$ remained significant predictors of PFS on multivariable analysis. KaplanMeier plots for OS and PFS stratified by significant multivariable predictors are shown in Figs. $4 \mathrm{C}-4 \mathrm{H}$.

\section{Discussion}

The study of MTTs, like any rare tumour type, has largely been limited to case reports in order to guide care. The highest level of evidence possible is a systematic review such as the one we have carried out. Prior to our report, a study by McConnell et al. in 2012, combined 99 adult and 25 paediatric MTT cases (1). With 124 adult cases, the current study provides a rigorous analysis of the updated literature. In addition to including a larger number of adult MTT cases, we restaged cases using the AJCC 8th edition staging system (which was feasible in all cases based on case descriptions) and examined the role of prior radiation exposure. Similarly, we found that margin status was associated with poorer PFS and adjuvant radiation with improved OS. In addition, we identified that positive NF1 status, prior radiation exposure and tumour location in the CNS or spine were also associated with poorer OS. In contrast to McConnell et al., tumour and nodal stage as well as increasing tumour size were not associated with survival on univariable or multivariable analysis(1). No survival benefit was observed for adjuvant chemotherapy.

NF1 and prior radiation exposure are well documented as risk factors for the development of MTT $(1,5)$. Out of the 124 cases in this review, 103 case reports commented on NF1 status, and $35.0 \%$ of these were positive for NF1. This is consistent with Li et al. who found $41.7 \%$ of their cases had NF1(4). McConnell et al. found NF1 status to be a negative prognostic factor with increased mortality on 
univariable analysis, however it did not remain significant on multivariable analysis(1). In this review, NF1 status was a significant predictor of both OS and PFS on univariable and multivariable analysis. LaFemina et al. examined 49 sporadic, 42 NF1-associated, and 14 radiation-induced MPNSTs(6). Interestingly, they found that sporadic MPNSTs and NF1-associated MPNSTs had similar survival and recurrence outcomes(6). This is not in keeping with our findings; however, MPNST subtypes were not reported in the analysis by LaFemina et al.(6) Given our findings, it is possible that NF1 associated MTTs behave more aggressively than other NF1 associated MPNSTs. In the current analysis, 5 cases of previous radiation exposure were identified, and it was found to be a significant predictor of worse OS on both univariable and multivariable analysis, which was consistent with LaFemina et al. reporting worse oncological outcomes for patients with RT-induced MPNSTs(6).

The mean age of MTT diagnosis in this review was 42.8 years. Previous literature states that NF1 positive patients tend to present as young males, and sporadic cases of MTT present more frequently in older females $(2,5)$. The mean age of male cases in our study population was 41.4 years, compared to 44.6 years for female cases. The majority of cases with a history of NF1 were male ( $n=26$, $72.2 \%$ ), with a mean age of 35.2 years, whereas females with NF1 ( $n=10,27.8 \%)$ had a slightly older mean age of 37.8 years. A study conducted by Terzic et al., found a mean age of 26.2 years (range: $3-44$ ) for their NF1 positive cases(5). This younger age of diagnosis for NF1 positive patients in the literature compared to the current study is largely attributed to the exclusion of paediatric cases.

Our systematic review provides a basis for treatment recommendations based on the management strategies and treatment outcomes observed. Almost all identified cases utilized surgery (95.9\%) suggesting that this modality is a cornerstone of treatment. Comparable to most solid malignancies, positive surgical margins were a poor prognostic factor highlighting the importance of planning for and obtaining an RO resection as was obtained in our case report. In this review, the most common tumour sites were head and neck and extremities. This is in keeping with other studies which found these tumour sites were common(2,4). CNS and spine tumour sites were significantly predictive of worse OS. This is likely due in part to difficulty resecting tumours in these sites as well as critical nearby organs limiting the potential adjuvant radiation dose.

Our study reinforces the critical importance of adjuvant radiotherapy. Radiotherapy was utilized in $60.7 \%$ of cases and was associated in significantly improved OS. Adjuvant radiotherapy was significant on univariable analysis for PFS, but it did not remain significant on multivariable analysis. Radiation dose varied, but most cases received doses greater than an equivalent dose in 2 Gy per fraction (EQD2) of $50 \mathrm{~Gy}$. In contrast, chemotherapy was not found to be of benefit and was even associated with worse PFS on univariable analysis. These findings are likely confounded by the heterogeneity of chemotherapy drugs and dosing schedules used, as well as a bias towards using chemotherapy in more advanced cases with adverse pathological features. Likely more active agents are needed to improve the poor survival observed in our cohort.

Although recent studies suggest that immunotherapy may be effective in slowing progression of plexiform neurofibromas in NF(7), the role of immunotherapy in MPNSTs is unknown. There are case reports indicating that immunotherapy may provide some benefit(8); however, we do not yet have any high-level evidence to support its use. Fortunately, there are currently several clinical trials underway examining this question. An active phase 2 trial examining the effects of pembrolizumab (anti-PD-L1 antibody) in MPNST patients that do not qualify for curative surgery is currently recruiting with results expected in 2022 (NCT02691026)(9). A second phase 2 trial is examining the effectiveness of nivolumab (anti-PD-L1 antibody) and ipilimumab (anti-CTLA-4 antibody) in treating a wide variety of rare tumours and has closed to accrual for many of the tumour types including MPNST, with results expected in 2021 (NCT02834013)(10). A third study of 20 patients with unresectable or refractory MPNST's (NCT02008877), treated with a combination of ganetespib and sirolimus was well tolerated but ineffective and did not meet criteria for further study(11).

Interestingly, T stage was not a significant predictor for either OS or PFS. This could be partially attributed to over $70 \%$ of our sample size presented at stage T3 or T4, or that tumour size may not be a factor that impacts the likelihood of local, regional or distant spread. In our review, only 4 cases had nodal disease and 1 patient had metastatic disease on presentation. $\mathrm{N}$ stage was also not a significant predictor for either OS or PFS, most likely due to the limited number of cases presenting with nodal involvement. This is in keeping with the literature that highlights presentations of MTT with nodal involvement or metastasis are rare $(5,12)$. Divergent differentiation was present in $24(19.8 \%)$ of the 121 cases in which it was reported. The most common types were chondroid ( $n=5,20.8 \%)$, osteocartilaginous $(n=5,20.8 \%)$ and glandular $(n=4,16.7 \%)$. Divergent differentiation was a significant predictor of worse OS on univariable analysis but did not remain significant on multivariable analysis.

Median and 5-year PFS was 8 months and 21.3\%, respectively. Just over half of the cases in this study experienced recurrence of the neoplasm $(n=63,50.8 \%)$, with the most common recurrence patterns being local only $(n=24,38.1 \%)$, distant only $(n=23,36.5 \%)$ and 
combined local and distant recurrences $(n=12,19.1 \%)$. This is similar to McConnell et al., where $50 \%$ of their cases had a recurrence and the median PFS was 6 months(1).

Systematic reviews are inherently limited by the data which is obtainable only through literature review. The majority of the studies used for this review were case reports, allowing for more detailed data collection; however, there were still missing data for many of the cases. Further, there was a high risk of publication bias across the studies included in this review due to their nature as case reports, as there is likely an over-representation of complex cases or impressive treatment responses, as these were more likely to have been written as case reports. Despite these limitations, for rare tumours such as MTTs where randomized clinical studies are not feasible, systematic reviews provide clinicians with evidence-based prognostic data and treatment recommendations for their patients.

\section{Conclusion}

We have completed a rigorous systematic review of adult MTTs, a very rare aggressive form of MPNST. Our results provide clinicians with information that they can use to determine specific prognostic factors and can provide their patients information on estimated oS and PFS. Based on our findings, we recommend that clinicians treat MTTs with radical excision, aiming for negative margins and that patients receive adjuvant radiotherapy.

\section{List Of Abbreviations}

Malignant triton tumours (MTTs)

Malignant peripheral nerve sheath tumours (MPNSTs)

Overall survival (OS)

Progression-free survival (PFS)

Hazard ratio (HR)

Confidence interval (Cl)

Neurofibromatosis 1 (NF1)

Magnetic resonance imaging (MRI)

Computed tomography (CT)

$\mathrm{R} 0$ resection (resection with negative margins)

American Joint Committee on Cancer [AJCC]

High power field (HPF)

Central nervous system (CNS)

\section{Declarations}

\section{Ethics Approval and Consent to Participate}

Not applicable

\section{Consent for Publication}

Informed consent was obtained from the patient for publication of this case report.

\section{Availability of Data and Materials}


The data supporting this systematic review are from previously reported case studies and datasets, which have been cited. The processed data are available from the corresponding author upon request.

\section{Competing Interests}

The authors declare that there is no conflict of interest regarding the publication of this paper.

\section{Funding}

No funding was applied for or received for this study.

\section{Author's Contributions}

The search strategy was created by AEP, LC, GB and ACN. Abstract review was completed by AEP and LC. Full text review was completed by AEP, AB and LC. AEP wrote the manuscript. ACN and DAP oversaw the project. Statistical analysis was performed by AW. All authors contributed to the final manuscript.

\section{References}

1. McConnell YJ, Giacomantonio CA. Malignant triton tumors-complete surgical resection and adjuvant radiotherapy associated with improved survival. J Surg Oncol. 2012;106(1):51-6.

2. Li Z, Xiang J, Yan S, Gao F, Zheng S. Malignant triton tumor of the retroperitoneum: a case report and review of the literature. World J Surg Oncol. 2012 May 30;10:96-96.

3. Woodruff J, Chernik N, Smith M, Millett W, Foote JRF. Peripheral nerve tumors with rhabdomyosarcomatous differentiation (malignant "triton" tumors). Cancer. 1973;32(2):426-39.

4. Li G, Liu C, Liu Y, Xu F, Su Z, Wang Y, et al. Analysis of clinical features and prognosis of malignant triton tumor: A report of two cases and literature review. Oncol Lett. 2015/09/29 ed. 2015 Dec;10(6):3551-6.

5. Terzic A, Bode B, Gratz KW, Stoeckli SJ. Prognostic factors for the malignant triton tumor of the head and neck. Head Neck. 2009;31(5):679-88.

6. LaFemina J, Qin L-X, Moraco NH, Antonescu CR, Fields RC, Crago AM, et al. Oncologic outcomes of sporadic, neurofibromatosisassociated, and radiation-induced malignant peripheral nerve sheath tumors. Ann Surg Oncol. 2012/08/10 ed. 2013 Jan;20(1):6672.

7. Gross A, Dombi E, Widemann B. Current status of MEK inhibitors in the treatment of plexiform neurofibromas [published online ahead of print, 2020 Jun 30]. Childs Nerv Syst. 2020;10.

8. Özdemir B, Bohanes P, Bisig B, Missiaglia E, Tsantoulis P, Coukos G, et al. Deep Response to Anti-PD-1 Therapy of Metastatic Neurofibromatosis Type 1-Associated Malignant Peripheral Nerve Sheath Tumor With CD274/PD-L1 Amplification. JCO Precis Oncol. 2019;1-6.

9. ClinicalTrials.gov. A Study of Pembrolizumab in Patients With Malignant Peripheral Nerve Sheath Tumor (MPNST), Not Eligible for Curative Surgery. Identifier NCT02691026. Natl Libr Med US. 2016 Jun;

10. ClinicalTrials.gov. Nivolumab and Ipilimumab in Treating Patients With Rare Tumors. Identifier NCT02834013. Natl Libr Med US. 2016 Jul 13;

11. Kim A, Lu Y, Okuno S. Targeting Refractory Sarcomas and Malignant Peripheral Nerve Sheath Tumors in a Phase I/II Study of Sirolimus in Combination with Ganetespib (SARC023). Sarcoma. 2020 Jan 30;

12. Tian L, Shang H-T, Bilal S, Li Y, Feng Z, Lei D, et al. Treatment of Malignant Triton Tumor in Zygomatic Region. J Craniofac Surg [Internet]. 2012;23(3). Available from:

https://journals.Iww.com/jcraniofacialsurgery/Fulltext/2012/05000/Treatment_of_Malignant_Triton_Tumor_in_Zygomatic.127.aspx

\section{Figures}




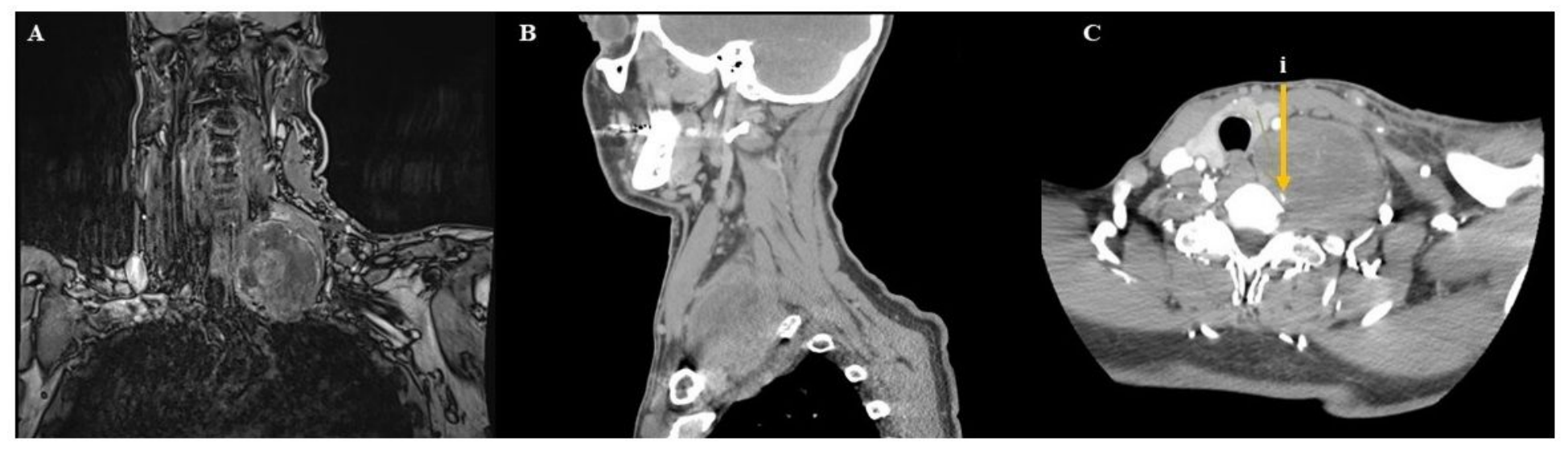

\section{Figure 1}

Magnetic resonance (MR) and computed tomography (CT) images. (A) MR image using T1-weighted post-gadolinium. Dixon opposed phase. Image in the coronal plane showing evidence of brachial plexus involvement. (B) CT image in the sagittal plane showing evidence of rib involvement. (C) CT image in the axial plane, (i) showing the encased left vertebral artery.

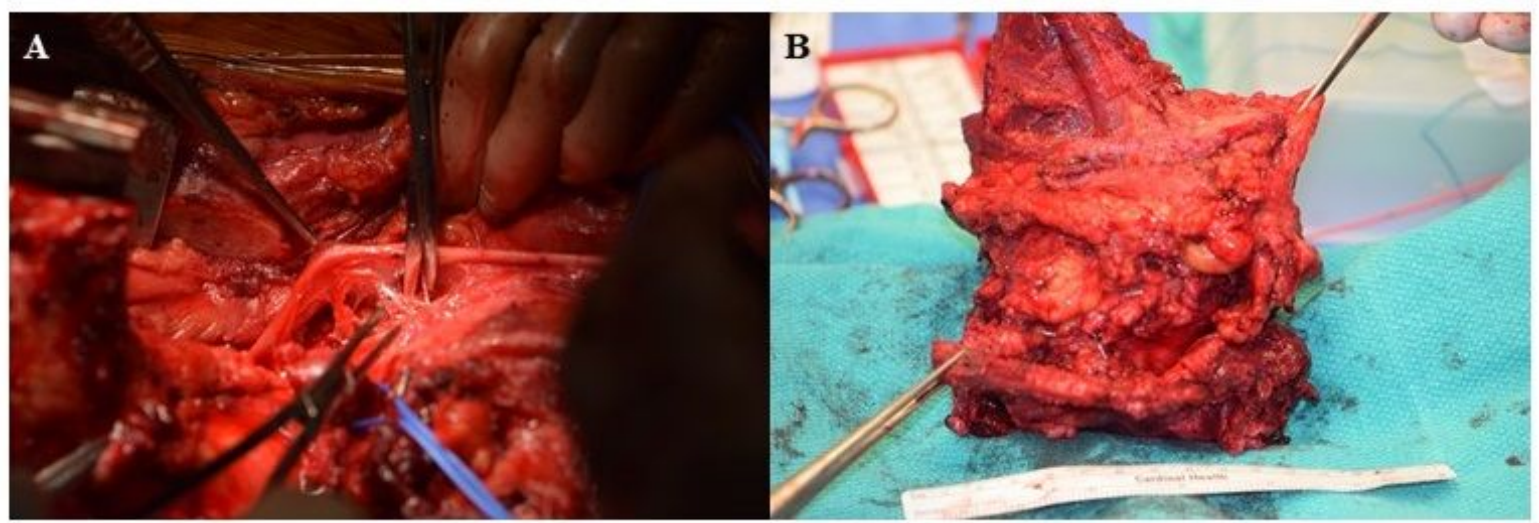

\section{Figure 2}

Intraoperative images. (A) Resection of tumour from the brachial plexus. (B) Final resected specimen. 


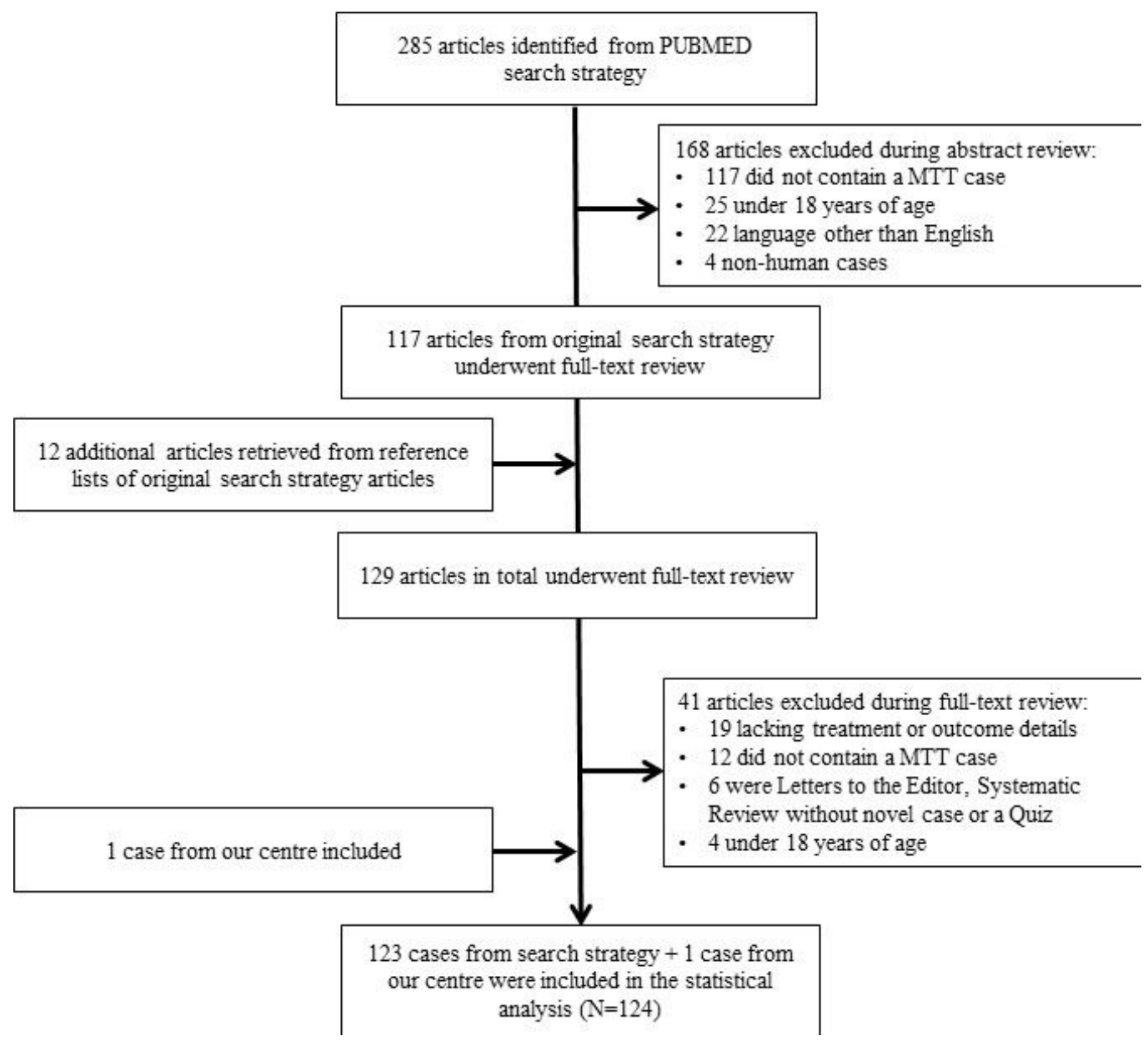

\section{Figure 3}

Results from search strategy 

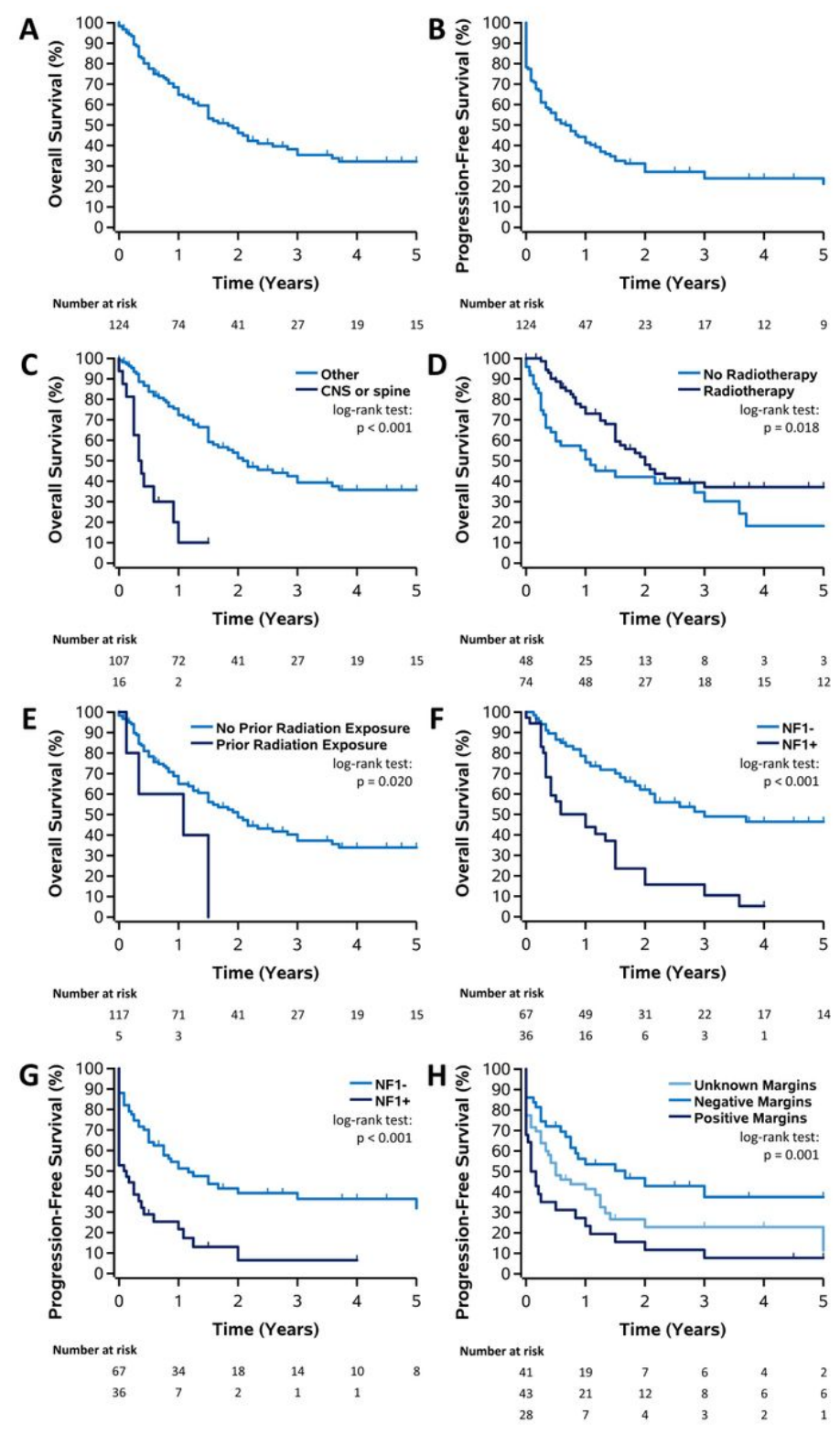

\section{Figure 4}

Kaplan-Meier plots for overall survival (OS) and progression-free survival (PFS) for all patients ( $n=124)$, and stratified by significant multivariable predictors of survival: (A) OS for all patients, (B) PFS for all patients, (C) OS by CNS or spine vs. other tumour site, (D) OS by adjuvant radiotherapy (yes vs. no), (E) OS by prior radiation exposure (yes vs. no), (F) OS by NF1 status (positive vs. negative), (G) PFS by NF1 status, and (H) PFS by surgical margin status (positive vs. negative vs. unknown).

\section{Supplementary Files}

This is a list of supplementary files associated with this preprint. Click to download.

- AdditionalFile1.pdf

- AdditionalFile2.pdf

- PRISMAChecklistBMC.pdf 